\title{
Sustained and transient gray matter volume changes after $n$-back training: A VBM study
}

\author{
Anna Miró-Padilla ${ }^{\text {a, }}$, Elisenda Bueichekú ${ }^{a}$, Jesús Adrián-Ventura ${ }^{\mathrm{a}}$, Víctor Costumero ${ }^{\mathrm{b}}$, \\ María-Ángeles Palomar-García ${ }^{a}$, Esteban Villar-Rodríguez ${ }^{a}$, Lidón Marin-Marin ${ }^{\text {a }}$, \\ Naiara Aguirre ${ }^{a}$, César Ávila ${ }^{a}$ \\ ${ }^{a}$ Neuropsychology and Functional Neuroimaging Group, Department of Basic Psychology, Clinical Psychology and Psychobiology, Universitat Jaume I, Castelló de la
Plana, Spain \\ ${ }^{\mathrm{b}}$ Center for Brain and Cognition, Pompeu Fabra University, Barcelona, Spain
}

\section{A R T I C L E I N F O}

\section{Keywords:}

Brain plasticity

CAT12

Gray matter changes

$N$-back

Voxel-based morphometry

Working memory training

\begin{abstract}
A B S T R A C T
Working memory training causes functional adaptations in the brain, which include changes in activation and functional connectivity that remain stable over time. Few studies have investigated gray matter (GM) changes after working memory training, and they have produced heterogeneous results without clarifying the stable effects of training. The present study was designed to test for sustained and transient anatomic changes after only $200 \mathrm{~min}$ of working memory training. The voxel-based morphometry technique was used in order to investigate the GM changes produced by a brief single $n$-back training, immediately and 5 weeks after finishing it. The sample was composed by 59 human participants who underwent MRI scanning and were assigned to either a training group or a passive control group. Results showed sustained GM volume enlargement in the right superior parietal cortex and a transient GM decrease in the right putamen. The brain adaptation in the right superior parietal cortex was stronger in individuals who showed greater improvements in performance. The results provide further evidence that a brief working memory training is able to produce brain plasticity in structures related to the trained task.
\end{abstract}

\section{Introduction}

Numerous behavioral studies have demonstrated that working memory training leads to improvements on the trained tasks (Klingberg, 2010). Neuroimaging investigations using MRI, which have been less extensive, have provided evidence of different cerebral modifications after working memory training. Activation changes in working memory brain areas have been reported, as well as an enhancement of the functional connectivity between them during the task and even in a resting-state (Constantinidis \& Klingberg, 2016). However, less is known about local anatomic changes after training. The present manuscript aims to investigate whether a brief working memory training leads to immediate and stable changes in gray matter (GM) volume in areas previously related to task performance.
As has been well-established, the $n$-back is the most widely used task in neuroimaging studies of working memory training. Both the single and dual versions of the task have been used in training designs, and both have shown their efficacy in improving the accuracy and time reactions in the trained participants (Jaeggi, Buschkuehl, Perrig, \& Meier, 2010; Küper \& Karbach, 2016; Soveri, Antfolk, Karlsson, Salo, \& Laine, 2017). In short training periods, single $n$-back was more effective than dual (Küper \& Karbach, 2016). After working memory training, the activation changes were usually present in brain areas that were already activated during the working memory task before training (Constantinidis \& Klingberg, 2016). The association areas of the prefrontal cortex (i.e. superior middle frontal cortex (BA 6) or dorsolateral prefrontal cortex (DLPFC; BA 9, 46)) and parietal cortex (i.e. posterior parietal cortex $(\mathrm{BA} 7,40)$ ) are the most consistently involved areas after

\footnotetext{
* Corresponding author at: Basic Psychology, Clinical Psychology and Psychobiology, Universitat Jaume I, Avda. Sos Baynat, s/n. E-12071, Castelló de la Plana, Spain.

E-mail addresses: amiro@uji.es (A. Miró-Padilla), bueichek@uji.es (E. Bueichekú), jadrian@uji.es (J. Adrián-Ventura), vcostume@uji.es (V. Costumero),

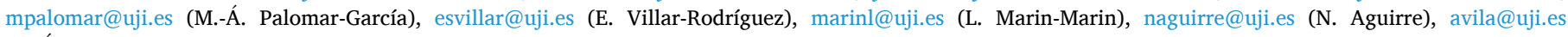
(C. Ávila).
} 
training, in most cases showing a reduced contribution to task performance after training (see Buschkuehl, Jaeggi, \& Jonides, 2012, for a review). Literature also suggests that working memory training increases functional connectivity between the prefrontal and parietal cortex, improving the capacity of a stimulus-encoding network (Durstewitz, Seamans, \& Sejnowski, 2000; Edin et al., 2009). All this evidence suggests that intense working memory practice increases the integrity and efficiency of frontoparietal networks. A second focus of interest is the striatum and the dopaminergic system. Several studies have described increases in the activation of the striatum (specifically in the putamen) linked to working memory training (Dahlin, Neely, Larsson, Bäckman, \& Nyberg, 2008; McNab \& Klingberg, 2008; Salminen, Kühn, Frensch, \& Schubert, 2016), with an inhibitory role that helps in the regulation of information flow (Kühn et al., 2013; McNab \& Klingberg, 2008). In addition, studies using positron emission tomography (PET) have demonstrated changes in the dopaminergic system as a result of this training (Backman et al., 2011; McNab et al., 2009). According to Constantinidis and Klingberg (2016), the role of the striatum does not seem to be crucial in working memory functions, but it could serve to facilitate plasticity in frontoparietal networks. This role of the striatum is especially developed during the first week of training (Kühn et al., 2013).

If there are activation and functional connectivity changes after training, it seems reasonable to hypothesize that they may be accompanied by structural changes in related brain areas. Prior studies have demonstrated training-related anatomic changes in other cognitive and motor domains. Specifically, increased GM volume or white matter density in diverse cerebral areas has been observed after training in physical skills (i.e. juggling) (Draganski et al., 2004), language (Golestani, Paus, \& Zatorre, 2002; Golestani \& Pallier, 2006; Ilg et al., 2008; Mårtensson et al., 2012; Stein et al., 2012), memory (Draganski et al., 2006; Engvig et al., 2010), or music skills (Bengtsson et al., 2005; Gaser \& Schlaug, 2003a \& 2003b; Hyde et al., 2009; Palomar-García, Zatorre, Ventura-Campos, Bueichekú, \& Ávila, 2017; Sluming et al., 2002). Cerebral areas affected by the training vary depending on the ability trained, ranging from, for example, the mid-temporal area and the left posterior intraparietal sulcus in juggling (Draganski et al., 2006) to the left insula/prefrontal cortex, the inferior parietal cortex (Golestani \& Pallier, 2007), and the hippocampus (Mårtensson et al., 2012) in language.

Regarding structural changes specifically after working memory training, research has been scarce. A review reported that working memory capacity positively correlated with regional GM and white matter volume in frontal and parietal regions in different clinical populations (i.e. stroke or multiple sclerosis) (Takeuchi, Taki, \& Kawashima, 2010). Longitudinal studies that employed morphometry and cortical thickness measures have not obtained a common pattern of results (Brooks et al., 2016; Colom et al., 2016; Lawlor-Savage, Clark, \& Goghari, 2019; Metzler-Baddeley, Caeyenberghs, Foley, \& Jones, 2016; Takeuchi et al., 2011). For example, Takeuchi and colleagues used voxel-based morphometry (VBM) to investigate structural changes in the brain after about $20 \mathrm{~h}$ of working memory training. After all that specific training, they found decreases in GM volume in the bilateral DLPFC, bilateral parietal lobe, and left superior temporal gyrus. However, they did not find relationships between the frontoparietal clusters and performance (Takeuchi et al., 2011). Another investigation also utilized VBM to study structural changes after working memory practice in a group of methamphetamine (MA) ex-abusers. The MA group was divided into two: one received treatment as usual (TAU) and the other received TAU and, additionally, trained 5 times a week for 4 weeks on an $n$-back task. Results yielded increased GM volume in the bilateral basal ganglia and reduced GM in the bilateral cerebellum in the group that performed the $n$-back training (Brooks et al., 2016). Furthermore, Colom and colleagues (2016), also using VBM, found increased GM volume in the left posterior cingulate cortex, the right cerebellum, and the right temporal lobe after an adaptive dual $n$-back training. They compared a trained group that completed 12 weeks ( 30 min twice a week) of dual $n$ back training with a passive control group. However, these authors failed to relate the psychological improvements in the training group with the structural GM changes (Colom et al., 2016). The use of different procedures and methodological strategies may explain these differences. Moreover, a general gap in longitudinal training studies involves the need to determine the stability of structural changes over time.

By means of a longitudinal VBM study, this work analyzed the brains of a sample of healthy adults before training, after 4 days of training on an adaptive version of single $n$-back training, and five weeks after finishing the training. Our previous study showed that this training improved $n$-back performance, in terms of accuracy and response speed, until 5 weeks after training, and it was accompanied by a decrease in the activation in frontoparietal areas (Miró-Padilla et al., 2019, 2020). Obtaining MRI structural data at three time points would allow us to investigate sustained and transient GM volume changes. We hypothesized that sustained GM changes would be present in frontoparietal areas as a result of the continuous use of these cortical areas during training and the maintenance of behavioral improvements. Moreover, transient changes after training would be observed in the striatum due to its role in facilitating cortical plasticity, even though this structure does not directly participate in working memory processes or in its improvement (e.g., the striatum is not involved in information maintenance / updating).

\section{Methods}

\subsection{Participants}

In the present study, 59 healthy right-handed individuals (20 male) participated. All were recruited from the student population of the Universitat Jaume I, and none of them reported a previous psychiatric or neurological diagnosis. The Ethical Committee of Universitat Jaume I approved the research project, and each subject gave his/her informed consent before participation. For their active participation, they received monetary compensation. Participants were randomly assigned to either an experimental condition (trained group) ( $\mathrm{N}=30$, mean age $=22.97 \pm 1.5,13 \mathrm{men}$ ) or a control condition (control group) $(\mathrm{N}=29$, mean age $=22.55 \pm 1.4,12$ men). The Matrix Reasoning Test (Wechsler Adult Intelligence Scale (WAIS) III-R) was used to evaluate their intellectual level (trained group: mean $=21.07 \pm 3.24$; control group: mean $=21.72 \pm 1.98$ ). Between-group t-student tests (or two sample $t$-test) were made in order to assess differences in gender distribution, age, and IQ. Results showed that differences were non-significant.

\subsection{Experimental design}

The experimental design (Fig. 1) used in the present work was reported in Miró-Padilla et al. (2019), which was focused on studying behavioral and task-fMRI brain changes produced by the trained task ( $n$ back). Three identical fMRI sessions were performed by both groups with the same adapted block-design $n$-back task. The trained group trained for $200 \mathrm{~min}$ on an adaptive $n$-back task between the two first scanner sessions. The first training session took place two days after the first fMRI session. The control group did not perform any cognitive or motor training. The groups did not have any training between the two last sessions. The pre-training session, post-training session, and followup session corresponded to Session 1 (S1), Session 2 (S2), and Session 3 (S3), respectively. Letters were used as visual stimuli, presented on the computer with the professional version 2.0 of E-Prime software (Psychology Software Tools, Pittsburgh, PA), installed in a Hewlett-Packard portable workstation (screen-resolution $800 \times 600$, refresh rate of 60 Hz). Through MRI-compatible goggles (VisuaStim, Resonance Technology, Inc., Northridge, CA, USA), subjects watched the laptop screen, and their responses were collected via MRI-compatible response-grips (NordicNeuroLab, Bergen, Norway). 


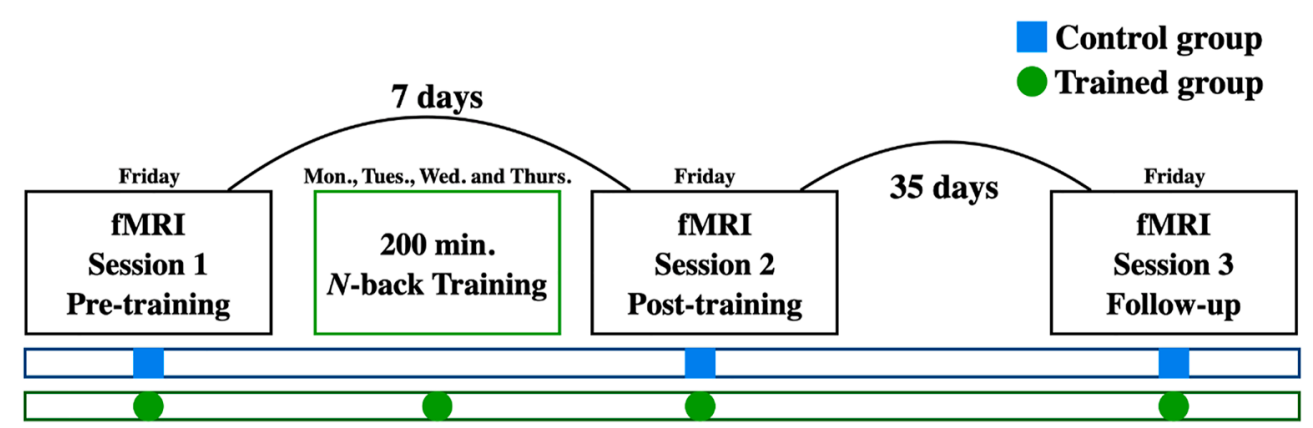

Fig. 1. Schematic description of the organization of the experiment. The experiment was performed by both groups equally in the three fMRI sessions. In the three fMRI scans, the anatomical data and task-fMRI acquisition data were acquired. Between the first two fMRI sessions, the trained group completed 4 sessions, a total of $200 \mathrm{~min}$, of a training task outside the scanner, and the control group did nothing. Training consisted of an adaptive single $n$-back task. Training group data correspond to the green circles, and control group data correspond to the blue squares.

\subsubsection{N-back fMRI task}

Three load levels were used to present the task: two working memory blocks (2-back and 3-back) and a baseline control task (0-back). Participants responded "yes" on the 0-back when the letter X (the target) appeared on the screen and "no" when there were no targets. On the working memory blocks, when the current letter shown on the screen matched the one presented 2 or 3 items back, subjects responded "yes"; and they responded "no" to any other letters. Using their right hand, participants gave manual responses, responding to targets with their thumb and to non-targets with their forefinger. The E-Prime's logfile saved each participant's accuracy and reaction time (RT) to each stimulus.

\subsection{2. $N$-back training task}

Four consecutive training sessions (TS) of single $n$-back were conducted by the trained group after fMRI S1 in our laboratory located at the university. Participants performed only one TS per day that lasted $60 \mathrm{~min}$ and was distributed in two phases. In the first phase, participants performed an adaptive $n$-back paradigm adapted from Jaeggi, Buschkuehl, Jonides, and Perrig (2008) for $50 \mathrm{~min}$, whereas in the second part, they performed a simple $n$-back task that lasted $10 \mathrm{~min}$. Their results on this test were useful for evaluating their progress on $n$-back. Therefore, the total training time was approximately $200 \mathrm{~min}$, plus 40 $\mathrm{min}$ for the second part. We used the same laptop as in the fMRI sessions, with the same display features and the same hardware for manual responses. Further details about the $n$-back fMRI and the training task design are reported in our previous study (Miró-Padilla et al., 2019) and can be found in the Supplementary Information.

\subsubsection{Accuracy and RT analyses}

IBM SPSS Statistics software (version 25) was utilized to process the behavioral data: accuracy (correct answers - false alarms) and RTs for participants' performance. An analysis of variance $(2 \times 3 \times 3$ ANOVA) with Group (training $\times$ control) as the between- subjects factor and Load Level (0-back vs. 2 -back vs. 3-back) and Session (1 vs. 2 vs. 3 ) as withinsubject factors was performed. With the data from the testing section of the training, a repeated-measures $2 \times 4$ ANOVA was performed, and Load Level (2-back vs. 3-back) and Training Session (1 vs. 2 vs. 3 vs. 4) were used as within-subject factors. The statistical significance threshold for the analysis was set at $\mathrm{p}<.05$.

\subsection{Neuroimaging data acquisition}

The same sequences were used in the three sessions, performed with a 1.5 T Siemens Symphony scanner (Erlangen, Germany), in order to collect the anatomical and functional MRI data. First, a high-resolution structural T1-weighted MPRAGE sequence was acquired (TR $=2200 \mathrm{~ms}$; $\mathrm{TE}=3 \mathrm{~ms}$; flip angle $90^{\circ}$, matrix $=256 \times 256 \times 160$; voxel size $=1 \times 1$ $\times 1 \mathrm{~mm}$ ). Finally, for task-fMRI ( $n$-back), a gradient-echo T2*-weighted echo-planar MR sequence covering the entire brain was used (TR/TE $=$ $2500 / 49 \mathrm{~ms}$, matrix $=64 \times 64 \times 28$, flip angle $=90^{\circ}$, voxel size $=3.5 \times$ $3.5 \times 4.48$; slice thickness $=4 \mathrm{~mm}$; slice gap $=0.48 \mathrm{~mm}$ ). A total of 260 volumes were recorded for $n$-back. All the scanner acquisitions were performed in parallel to the anterior commissure-posterior commissure plane (AC-PC), and they covered the entire brain. Participants were placed in a supine position in the MRI scanner, and their heads were immobilized with cushions to reduce involuntary motion. Furthermore, participants were asked to minimize head movement, even while giving the answers.

\subsection{Neuroimaging analysis}

\subsubsection{Voxel-based morphometry preprocessing}

Voxel-based morphometry (VBM) was performed with the Computational Anatomy Toolbox (CAT12; v1184; http://dbm.neuro.uni-jena. de/cat/) for the Statistical Parametric Mapping (SPM12) package (v6906; Wellcome Department of Imaging Neuroscience, London, England; www.fil.ion.ucl.ac.uk/spm/) in the MatLab 2015b environment (www.mathworks.com). First, each subject's anatomical image was reoriented to the AC-PC plane to have the same point of origin (anterior commissure). Then, the standard preprocessing procedure using the Segment Longitudinal Data module in the CAT12 manual was performed. The analysis of the longitudinal data requires customized processing that takes into account the characteristics of the intra-subject analysis. The preprocessing included: 1) registration to the mean image for each subject by an inverse-consistent realignment; 2) registration to a standard template provided by the International Consortium of Brain Mapping (ICBM); 3) segmentation of the images into GM, white matter, and cerebrospinal fluid; 4) DARTEL normalization of the GM segments to the Montreal Neurological Institute (MNI) template; and 5) modulation by the "affine + nonlinear" components derived from spatial normalization. After the preprocessing, the total intracranial volume (TIV) was estimated for each subject in each session, and a data quality check was carried out using a CAT12 toolbox (VBM data homogeneity) to assess the homogeneity of the GM tissues. No outliers were identified. Finally, images were spatially smoothed using an 8-mm FWHM Gaussian kernel.

\subsubsection{Voxel-based morphometry post-processing analysis}

For the VBM analysis, we used the CAT12 for all the statistical analyses of interest within the framework of the general linear model. For all analyses, age and TIV were included as covariates. Sex was not included in the analyses due to its high collinearity with TIV. The threshold masking used was 0.1 , whereas we also applied the SPM's non-stationary smooth correction. The GM volume of the control group was compared to that of the trained group across the sessions. Due to the non-directional nature of our hypotheses, the statistical inference in SPM analyses was two-sided. Following recent recommendations by Chen et al. (2019), we established an uncorrected voxel-level threshold at $\mathrm{p}<0.0005$. Then, FWE correction at the cluster-level was achieved by selecting those clusters with a $\mathrm{p}<0.025$ FWE. The FWE correction is based on the random field theory (RFT). All the statistical analyses were done at the whole-brain level, including cortical and subcortical areas, as well as, the cerebellum. First, S1 data were used to perform a two- 
sample $t$ test to examine the equality of the GM volume in the two groups, so that between-group brain differences found in subsequent sessions would not be due to preexisting differences. Then, longitudinal whole-brain analyses were performed, specifically using interaction analyses between sessions and groups to evaluate transient and sustained changes. On the one hand, transient changes due to training that were recovered in the long-term were investigated using a polynomial quadratic contrast (Trained group (S1, S3 vs. S2) vs. Control group (S1, S3 vs. S2)). On the other hand, two different requirements were used to determine sustained changes in GM volume due to training: 1) changes should be produced at S2 and must be observed in the S1 vs S2 contrast; 2 ) changes should be stable at $S 3$ and must be observed when comparing S1 to the average of S2 and S3 (Trained group (S1 vs. S2, S3) vs. Control group ( $S 1$ vs. S2, S3)). Stable changes will be observed when an overlap occurs between brain areas obtained in both contrasts.

In addition, the possible relationship between the improvement in the working memory task (i.e., improvement in accuracy and RTs) and the changes in GM volume was tested. The GM volume (in $\mathrm{ml}$ ) from the clusters of regions with statistically significant GM volume changes after the training was calculated at the individual level. To do so, the modulated GM volumes (without smooth) were obtained for each structure via a MatLab script (http://www0.cs.ucl.ac.uk/staff/g.ridgwa y/vbm/get_totals.m). To obtain the GM volume change, we calculated the difference in milliliters (ml) in the same way as in the aforementioned contrasts, that is, by comparing the GM volume of S1 to the average of S2 and S3 ([S1 - ((S2 + S3)/2)]) and the GM volume of S2 to the average of $S 1$ and $S 3([S 2-((S 1+S 3) / 2)])$ in the clusters with significant changes. Next, to study the brain-behavior relationships, linear bivariate Pearson's correlation analyses were conducted, using IBM SPSS Statistics (version 25), by taking the GM volumes extracted from the VBM's significant clusters and the improvement in the $n$-back fMRI and $n$-back training scores. To perform the correlation analyses with the GM volume, we obtained the $n$-back performance improvement by calculating the difference across sessions - from S1 to S2 and S3 - in mean accuracy (number of correct answers) and in RT averages. The following formulas were applied: [S2(or S3) - S1] for accuracy and [S1 S2(or S3)] for RT averages. In the case of the training data, we used: [TS4 - TS1] for accuracy and [TS1 - TS4)] for RT averages. The formula was applied three different times: with the averaged 2-back and 3-back levels together (named $n$-back accuracy or $n$-back RTs) and with 2-back and 3-back separately.

\section{Results}

The data, sample, and part of the methodology used in the present study are the same as in Miró-Padilla et al. (2019, 2020). The former study was focused on studying behavioral and task-fMRI brain changes due to the trained task (n-back), whereas the latter study focused on the transfer effects from the transfer task (PASAT). The behavioral results reported in the present analyses consist of additional participants that were not included in those prior analyses. Therefore, the behavioral results differ a little and have been explained accordingly.

\subsection{Accuracy and RT results}

\subsubsection{N-back behavioral fMRI results}

The analyses of variance were conducted separately for accuracy and for RTs. On the one hand, the accuracy analysis (Fig. 2) showed that all the participants improved their performance in S2 and S3, compared to $\mathrm{S} 1$, as indicated by the main effects for Session $\left(\mathrm{F}_{(2,55)}=28.76 \mathrm{p}<.001\right.$; $\left.\eta_{\mathrm{p}}^{2}=0.51\right)$ and Load Level $\left(\mathrm{F}_{(2,55)}=41.81 \mathrm{p}<.001 ; \eta_{\mathrm{p}}^{2}=0.6\right)$. These main effects were driven by significant Group $\times$ Session $\left(\mathrm{F}_{(2,55)}=\right.$ $\left.10.07 \mathrm{p}>.001 ; \eta_{\mathrm{p}}^{2}=0.27\right)$, Load Level $\times$ Session $\left(\mathrm{F}_{(4,53)}=9.92 \mathrm{p}>.001\right.$; $\left.\eta_{\mathrm{p}}^{2}=0.43\right)$, and Load Level $\times \operatorname{Group}\left(\mathrm{F}_{(2,55)}=5.39 \mathrm{p}=.007 ; \eta_{\mathrm{p}}^{2}=0.16\right)$ interactions. In the same way, the Load Level $\times$ Session $\times$ Group interaction reached significance $\left(\mathrm{F}_{(4,53)}=4.26 \mathrm{p}=.005 ; \eta_{\mathrm{p}}^{2}=0.24\right)$,

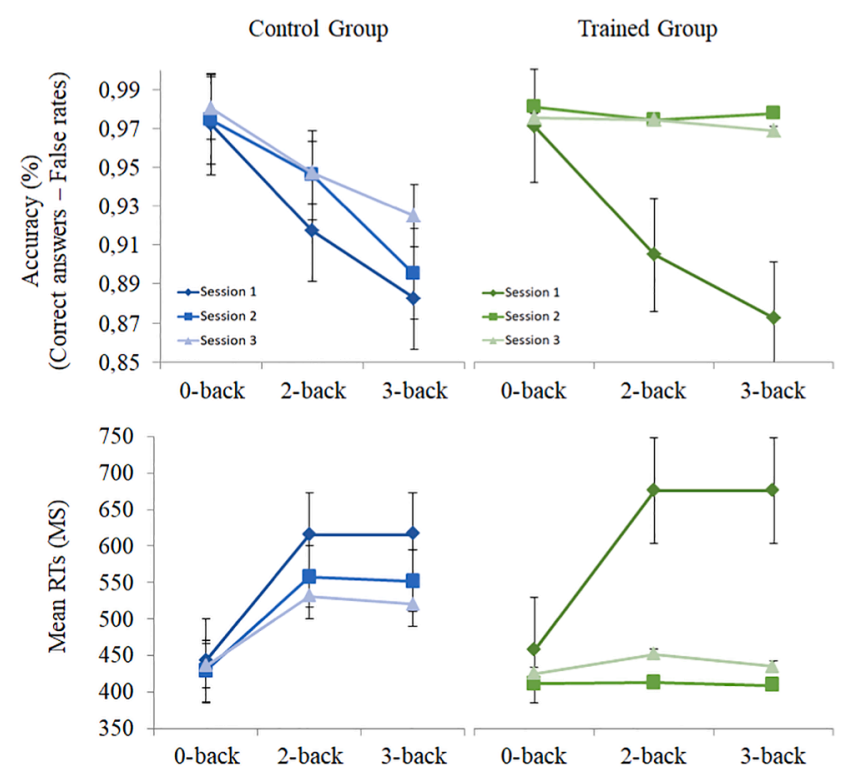

Fig. 2. Results of the $n$-back behavioral analysis. Accuracy (correct answers false alarms) and mean RTs (in milliseconds) per session have been plotted as a function of time. Pre-training session, post-training session, and follow-up session correspond to Session 1, Session 2, and Session 3, respectively. Training group data correspond to the green bars, and control group data to the blue bars. Error bars represent standard error.

indicating that the trained group became more accurate in S2 and S3 than the control group when performing the 2-back and 3-back load levels. On the other hand, the analysis of the RT scores (Fig. 2) also revealed significant main effects for Session $\left(\mathrm{F}_{(2,56)}=57.34 \mathrm{p}<.001 ; \eta_{\mathrm{p}}^{2}\right.$ $=0.67)$ and Load Level $\left(\mathrm{F}_{(2,56)}=81.13 \mathrm{p}<.001 ; \eta_{\mathrm{p}}^{2}=0.74\right)$. In $\mathrm{S} 2$ and $\mathrm{S} 3$, both groups responded faster, on average, than in S1. Moreover, both responded faster, on average, at the 0-back load level compared to the 2back load level, and faster, on average, at the 2-back load level compared to the 3-back load level. Significant two-way interactions were obtained for the Group $\times$ Session $\left(\mathrm{F}_{(2,56)}=31.91 \mathrm{p}<.001 ; \eta_{\mathrm{p}}^{2}=\right.$ 0.53), Group $\times$ Load Level $\left(\mathrm{F}_{(2,56)}=4.82 \mathrm{p}=.012 ; \eta_{\mathrm{p}}^{2}=0.15\right)$ and Load Level $\times$ Session $\left(\mathrm{F}_{(4,54)}=32.47 \mathrm{p}<.001 ; \eta_{\mathrm{p}}^{2}=0.71\right)$ interactions. Notably, all these significant effects were qualified by the three-way Load Level $\times$ Session $\times$ Group interaction, and the effect size observed for this interaction was large $\left(\mathrm{F}_{(4,54)}=11.01 \mathrm{p}<.001 ; \eta_{\mathrm{p}}^{2}=0.45\right)$. As expected, this interaction indicated that the training group, compared to the control group, was faster, on average, in S2 and S3 at the 2-back and 3-back load levels.

\subsection{2. $N$-back behavioral training results}

The accuracy and RTs (see Table 1 for means and standard deviations) of the test part of the training were used to perform a repeatedmeasures $2 \times 4$ ANOVA. Regarding accuracy, the analysis showed

Table 1

Mean (M) and standard deviations (SD) values for the accuracy scores (correct answers - false alarms) and for the RTs (in milliseconds) across the four training sessions performed by the Training group after each training session (these data were obtained from the $n$-back test task).

\begin{tabular}{|c|c|c|c|c|c|c|c|c|}
\hline \multirow[t]{3}{*}{ TS } & \multicolumn{4}{|c|}{ Accuracy } & \multicolumn{4}{|l|}{ RTs } \\
\hline & 2-back & & 3-back & & 2-back & & 3-back & \\
\hline & M & SD & M & SD & M & SD & M & SD \\
\hline 1 & 28.61 & 1.14 & 27.71 & 1.39 & 448.84 & 88 & 431.51 & 87.52 \\
\hline 2 & 28.76 & 1.03 & 28.04 & 1.61 & 402.90 & 56.33 & 397.04 & 76.8 \\
\hline 3 & 28.88 & 0.87 & 28.65 & 1.32 & 388.27 & 49.49 & 387.6 & 85.27 \\
\hline 4 & 29.08 & 0.99 & 28.52 & 1.58 & 380.33 & 48.11 & 383.59 & 75.33 \\
\hline
\end{tabular}

*TS = Training session; RTs = Reaction times; $\mathrm{M}=$ Mean; SD = Standard Deviation. 
significant effects for the training session $\left(\mathrm{F}_{(3,27)}=6.57 \mathrm{p}=.002 ; \eta_{\mathrm{p}}^{2}=\right.$ $0.42)$ and the $n$-back level or load $\left(\mathrm{F}_{(1,29)}=11.99 \mathrm{p}=.002 ; \eta_{\mathrm{p}}^{2}=0.29\right)$. As expected, the performance on the $n$-back task improved significantly and progressively after each TS at both the 2-back and 3-back levels. In the case of the RT values, results revealed a statistically significant effect of the training session $\left(\mathrm{F}_{(3,27)}=11.32 \mathrm{p}<.001 ; \eta_{\mathrm{p}}^{2}=0.56\right)$, indicating that participants' RTs, on average, declined significantly as the TS progressed. The overall results indicate that trained participants steadily improved their accuracy as they accumulated the $200 \mathrm{~min}$ of training.

\subsection{Voxel-based morphometry results}

The data collected in S1 were used to perform a two-sample $t$ test analysis in order to identify possible basal differences in GM volume between the two groups before training. The results showed no statistically significant differences. Consequently, we ruled out the possibility that differences between groups in subsequent sessions were due to preexisting differences. The threshold was $p<.025$ FWE, clustercorrected using a threshold of $\mathrm{p}<.0005$ at the uncorrected voxel level.

The sustained effects of training were determined from brain areas that were significant and overlapped after applying these two contrasts: $\mathrm{S} 1$ vs S2 and S1 vs S2, S3 ( $\mathrm{p}<0.025$ FWE cluster-corrected, in both cases). Results showed a stable increment in GM volume in the trained group in comparison with controls in the right superior parietal cortex (RSPC; Fig. 3AB). On the one hand, for the Trained group (S2 >S1) > Control group $(S 2>S 1)$ contrast, the MNI coordinates for the maximum were $\times=20, y=-859$, and $\mathrm{z}=69$, the Brodmann areas (BA) corresponded to 3, 2 and 5, and, lastly, the $\mathrm{Z}$ and $\mathrm{T}$ values were 5.02 and 5.34, respectively. On the other hand, for the Trained group $(S 1<S 2, S 3)>$ Control group $(S 1<S 2$, S3) contrast, the MNI coordinates for the maximum were $x=15, \mathrm{y}=-41$, and $\mathrm{z}=72, \mathrm{BA}=3,5$ and $7, \mathrm{Z}$-value $=$ 4.19 and $\mathrm{T}=4.38$. The reverse contrasts yielded no statistically significant effects.

Transient changes due to training were studied using a polynomial contrast. This analysis assessed the GM volume changes after training that did not remain stable. Therefore, increases or decreases in GM volume were sought, comparing the brain state right after training (S2) to the GM volume before training (S1) and five weeks after training (S3). The contrast Control group (S1, S3 > S2) > Trained group (S1, S3 > S2) yielded a significant difference in the right putamen (Fig. 3), consisting of a volume decrease in the trained group compared to the control group. In this case, the MNI coordinates for the maximum were $\times=39$, $\mathrm{y}=-26$, and $\mathrm{z}=5$, BA 48 , Z-value $=4.75$, and $\mathrm{T}=5.03$. The opposite contrast did not yield any statistically significant differences.

\subsection{Correlation analyses with performance}

The relationship between performance improvement (both accuracy and RTs) and the GM volume changes after training was studied by means of bivariate Pearson's correlation analysis. The brain regions that changed their GM volumes after training were used as regions of interest (ROIs): the RSPC for the stable change and the right putamen for the transient change. Finally, two variables were created representing the GM volume changes studied before: 1$)$ Stable change: [S1 - ((S2 + S3)/ 2)]; 2) Transient change: [S2 - ((S1 + S3)/2)].

On the one hand, the analysis of the sustained GM volume change found in the RSPC and the $n$-back fMRI improvements showed a significant positive correlation between the GM volume change in the RSPC and the improvement in RTs from S1 to S2 and from S1 to S3 in the trained group (see Fig. 4 for values). This significant correlation was not
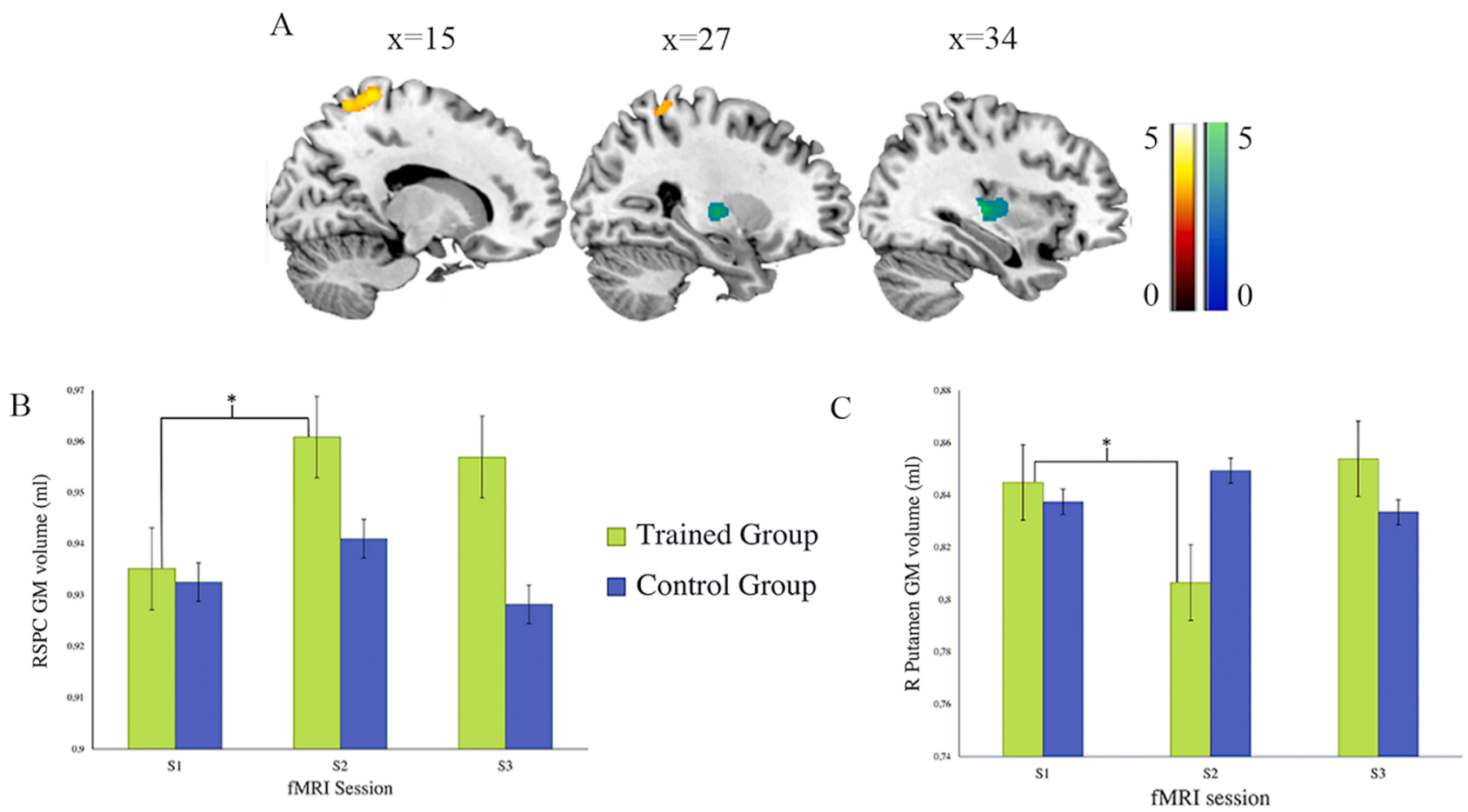

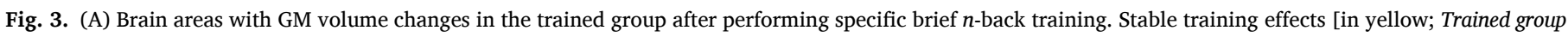

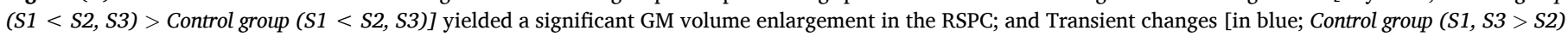

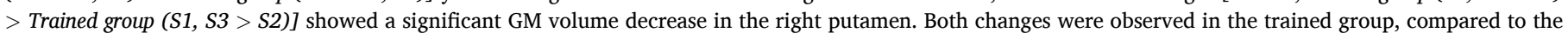

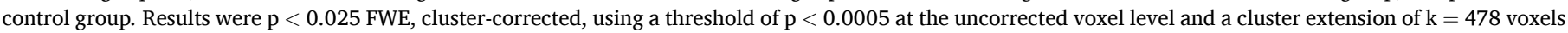

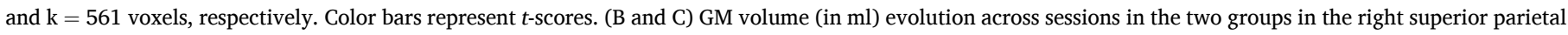

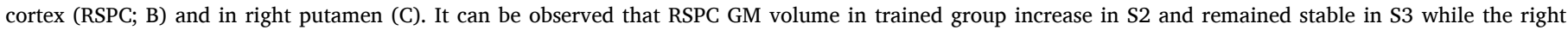

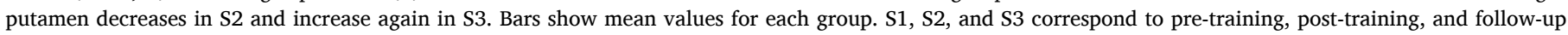

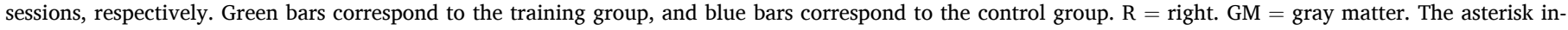
dicates statistically significant group differences. 


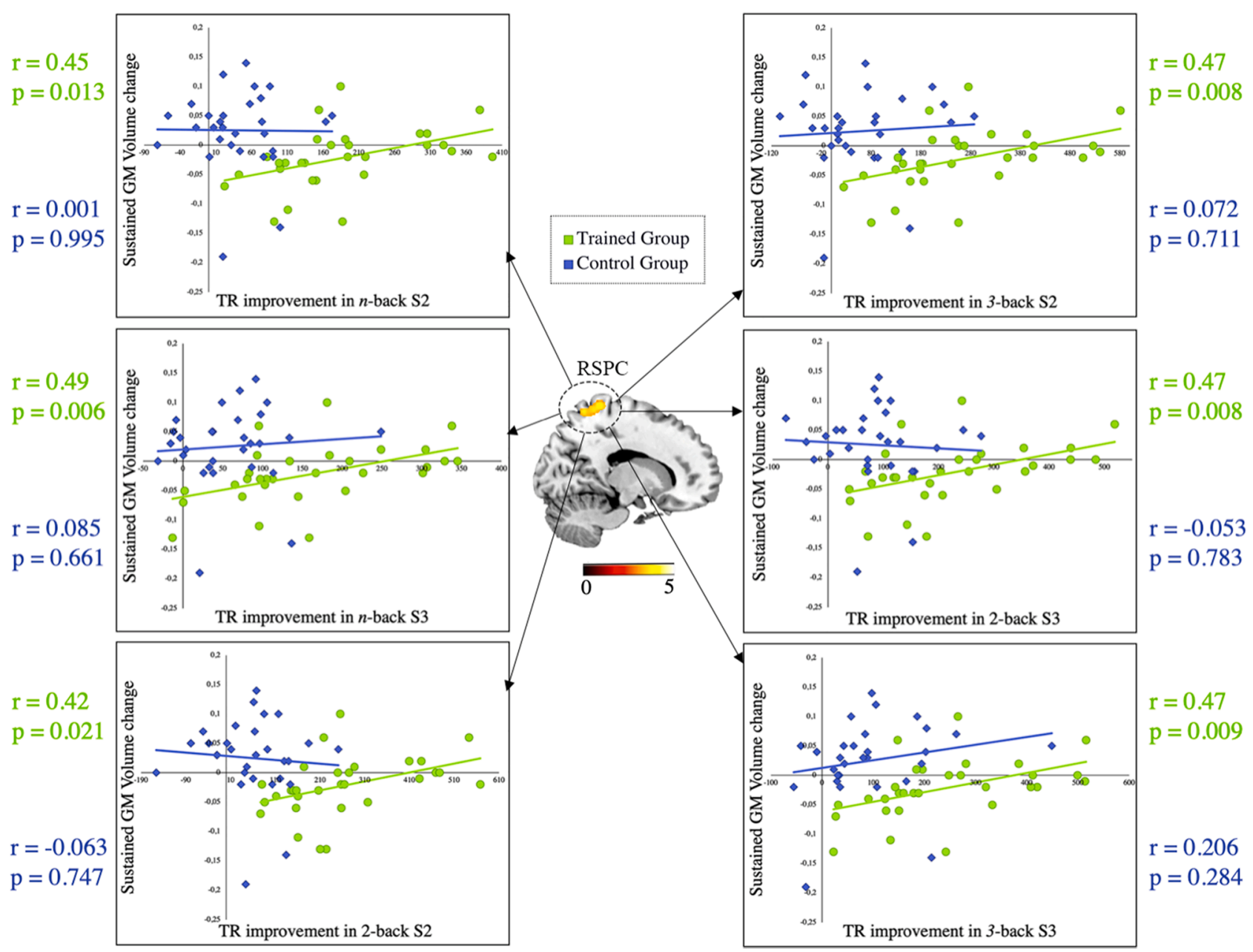

Fig. 4. $N$-back reaction time (RT) improvement in session 2 (S2) and session 3 (S3) related to the sustained gray matter (GM) volume change (in ml) in the right superior parietal cortex (RSPC). Sustained GM volume changes in RSPC were positively correlated with $n$-back RT improvement in the trained group. A color scale based on $t$ values is shown with yellow areas representing the sustained effect (i.e., regions with increased GM volume in trained participants; Trained group (S1 < S2, $S 3)>$ Control group $(S 1<S 2, S 3)$ ). In the scatter plots, the GM volume at RSPC is displayed for each subject, with circular green dots for the training group and blue diamond dots for the control group. The correlation coefficients and $\mathrm{P}$ values are presented next to each scatter plot.

found in the Control group. The difference between these two correlation coefficients was statistically significant in the first case (Trained group $>$ Control group, $\mathrm{z}=1.76 \mathrm{p}=.039$ one-tailed). On the other hand, the same significant positive relationship was found only in the trained group for RT improvements in S2 and S3, specifically at 2-back and 3back load levels (Fig. 4). At 2-back, the difference between these two correlation coefficients was statistically significant (Trained group $>$ Control group, $\mathrm{z}=1.86 \mathrm{p}=.031$ and $\mathrm{z}=2.05 \mathrm{p}=.02$ one-tailed). This means that participants with greater improvements in their RTs, responding more quickly in S2 and S3 than in S1, had more GM volume after $n$-back training in the RSPC. The sustained GM volume change in the RSPC also correlated with behavioral improvements in the $n$-back processing after training in the trained group. Analyses yielded a significant positive correlation between this area and the improvement in the last TS in: $n$-back accuracy $\left(\mathrm{r}_{25}=0.46 \mathrm{p}=.011\right), 2$-back accuracy $\left(\mathrm{r}_{25}=0.4 \mathrm{p}=.027\right), 3$-back accuracy $\left(\mathrm{r}_{25}=0.37 \mathrm{p}=.045\right)$, and 3-back RTs $\left(r_{25}=0.48 \mathrm{p}=.008\right)$. Finally, when analyzing the relationship between the cluster with decreased GM volume (transient changes) and task improvement, the analysis did not show any significant correlations between improvement on the task and the GM volume change in the right putamen.

\section{Discussion}

Having previously observed that an adaptive $200 \mathrm{~min}$ of single $n$ back training improves behavioral performance and causes cerebral modifications, as indicated by the decrease in the activation in diverse brain areas involved in working memory on the trained and the transfer task (Miró-Padilla et al., 2019, 2020), we extend our previous work by investigating the GM volume changes after $200 \mathrm{~min}$ of an adaptive single $n$-back training, using a new three-time point approach. In addition, the relationship between brain changes and behavioral improvement in task performance was investigated. Using the VBM method, we compared the GM volume of two groups (trained group and passive control group) before training, immediately after training, and after five weeks of no training. Using this new three-time point approach, we found two significant changes due to training. We first obtained a sustained increment in the GM volume of the right superior parietal cortex that positively correlated with performance improvement. We also found a transient reduction in GM volume in the right putamen. Our results demonstrate that a brief and intensive $n$-back training causes GM volume changes in brain areas involved in working memory processing.

As previously obtained in this sample, $n$-back training was associated with behavioral improvements and decreased brain activation in frontoparietal areas (Miró-Padilla et al., 2019, 2020). Previous results also showed a sustained decrease in the activation of bilateral frontal and 
right parietal areas (including the RSPC) after training. Thus, the functional response in the main frontoparietal areas presumably participated actively throughout the training process. By contrast, the basal ganglia did not participate significantly in the task at baseline, and they did not change their functional activity after training. In addition, we found a positive relationship between the volume changes in the RSPC and the improvements on the $n$-back in the trained group after training, thus linking the anatomic changes with the behavioral improvement. This relationship was not found in the control group.

Although GM changes in the frontal cortex were expected, in the present study no conclusive evidence was found in this cerebral region. However, consistent with our hypothesis, a sustained GM increase was found in the RSPC, a brain area with a prominent role in working memory. Functional changes in this area have been observed in comparable working memory training studies (Buschkuehl, HernandezGarcia, Jaeggi, Bernard, \& a, \& Jonides, 2014; Schneiders (2011)). Buschkuehl and colleagues (2014) also used a brief $n$-back training $(<3$ h) to observe the brain activation changes after the training. They reported a perfusion decrease in RSPC (BA 5) at rest in the post-training session, indicating that BA 5 is involved in working memory training and $n$-back task performance. The superior parietal cortex is not an essential area in the $n$-back task (Owen, McMillan, Laird, \& Bullmore, 2005). However, several studies have reported that BOLD activation in this area is a good positive predictor of behavioral performance (DeYoung, Shamosh, Green, Braver, \& Gray, 2009; Owens, Duda, Sweet, \& MacKillop, 2018; Zou et al., 2013), and that changes in BOLD activity were positively correlated with anatomic volume (Owens et al., 2018). This latter study, performed with more than 1000 individuals, additionally showed that this area had the strongest integration between BOLD activity during the task and GM volume. The role of this area in working memory has been related to spatial processing, but also to verbal and phonological stimuli (Collette, Hogge, Salmon, \& Van der Linden, 2006; Nee et al., 2013). Overall, the superior parietal lobe is critically important for top-down manipulation with a variety of types of information, as well as relevant to attention towards relevant items (Humphreys \& Lambon Ralph, 2015; Koenigs, Barbey, Postle, \& Grafman, 2009; Nee et al., 2013). Importantly, this increase in volume was greater in participants with more improvement in their RTs from S1 to S2 and S1 to S3. This result suggests that participants with greater improvement in their speed when performing the $n$-back had more GM volume in the RSPC after training. We tentatively interpret this result as indicating that participants who had more parietal GM volume changes had adapted their cerebral resources to improve on the task, showing greater RT improvement than other participants who did not change their GM as much.

The results of the present study also showed a transient reduction in the volume of the posterior putamen that was not observable five weeks later. Although not as important as frontoparietal areas, the striatum plays a role in working memory processing. Recent data suggest that this brain area may modulate individual differences in working memory capacity and the effect of training by facilitating plasticity in the cortex (Constantinidis \& Klingberg, 2016; McNab \& Klingberg, 2008). Several previous studies have provided clues to help to understand the role of the striatum in working memory. First, there are developmental studies showing that striatal activity during working memory tasks is a good predictor of future (but not current) working memory capacity (Darki \& Klingberg, 2015; Ullman, Almeida, \& Klingberg, 2014). Second, a PET study showed that working memory training enhanced dopamine release in the striatum, emphasizing the important role of transient cerebral processes related to striatal D2 receptor activity in working memory (Backman et al., 2011). Third, genetic studies showed the role of polymorphisms related to the DAT-1 transporter (Brehmer et al., 2009; Söderqvist et al., 2012) and DRD2 receptor expression (Söderqvist, Matsson, Peyrard-Janvid, Kere, \& Klingberg, 2014) in individual differences in improvements after working memory training. Finally, and consistent with data from the present study, the role of the striatum is more relevant in the first stages of training (Kühn et al., 2013). All of this evidence is consistent with a prominent role of the striatum in the initial stages of working memory training, probably facilitating plasticity in frontoparietal areas.

This neuroimaging study was developed in order to use a three-time point approach for the first time, allowing us to provide new information about neuroplasticity after a working memory training. Nevertheless, this study has a few limitations. Due to the lack of an active control group, these results should be interpreted with caution. The training group came to our laboratory on four consecutive days, and they had more contact with the experimenters than the control group did, which may result in motivational differences between the two groups in terms of task efficiency. In future studies, active control groups should be included in the study design because the observed gains may not be due to working memory training per se, but to the training in general.

In conclusion, the present results showed that our short cognitive training can cause plasticity in brain structures related to the trained task and linked to the behavioral improvement. Sustained changes were found in the right superior parietal cortex, and transient changes were observed in the right putamen, both structures closely related to working memory. Increased GM volume was found in the RSPC in the trained participants, and the reverse effect was seen in the right putamen. We reported stability in the RSPC changes after five weeks of no training, and the results pointed to a relationship between the GM volume changes and the behavioral improvement on the task. Along with this, empirical structural neuroimaging data were provided to understand the mechanisms of the effects of $n$-back working memory training. Moreover, the anatomical changes in neural areas associated with working memory reported here demonstrate that cognitive training has an effect on the brain and on brain measurements important for cognitive skillfulness.

\section{CRediT authorship contribution statement}

Anna Miró-Padilla: Conceptualization, Methodology, Formal analysis, Investigation, Writing - original draft, Visualization. Elisenda Bueichekú: Conceptualization, Methodology, Writing - review \& editing. Jesús Adrián-Ventura: Formal analysis, Writing - review \& editing. Víctor Costumero: Software. María-Ángeles Palomar-García: Investigation. Esteban Villar-Rodríguez: Data curation. Lidón MarinMarin: Investigation. Naiara Aguirre: Investigation. César Ávila: Conceptualization, Writing - review \& editing, Supervision, Project administration, Funding acquisition.

\section{Acknowledgments}

This work was supported by grants from Universitat Jaume I (P1•1B2013-63) and Ministerio de Economia y Competitividad (PSI2013-47504-R and PSI2016-78805-R). Authors J. Adrián-Ventura, E. Villar-Rodríguez, L. Marin-Marin and N. Aguirre were supported by a pre-doctoral graduate program grant (National FPU to J.A-V, L.M-M, E. V-R and N.A). In addition, this work was supported by a post-doctoral graduate program grant to V. Costumero, M-Á. Palomar-García and A. Miró-Padilla (Juan de la Cierva to V.C and postdoc-UJI to M-Á.P-G and A.M.P). Author E. Bueichekú was funded by a postdoctoral grant from the "Generalitat Valenciana (2018 APOSTD)" and the "European Social Fund (Investing in your future)".

\section{Additional information}

Conflict of interest: None.

Competing Interests: The authors declare no competing interests.

Ethical approval: The study was approved by the institutional Ethics Committee of the Universitat Jaume I.

Informed consent: All the participants provided written informed consent prior participation. 


\section{Appendix A. Supplementary data}

Supplementary data to this article can be found online at https://doi. org/10.1016/j.nlm.2020.107368.

\section{References}

Backman, L., Nyberg, L., Soveri, A., Johansson, J., Andersson, M., Dahlin, E., Rinne, J. O. (2011). Effects of Working-Memory Training on Striatal Dopamine Release, 718718 Science, 333(6043). https://doi.org/10.1126/science.1204978.

Bengtsson, S. L., Nagy, Z., Skare, S., Forsman, L., Forssberg, H., \& Ullén, F. (2005). Extensive piano practicing has regionally specific effects on white matter development. Nature Neuroscience, 8, 1148. https://doi.org/10.1038/nn1516.

Brehmer, Y., Westerberg, H., Bellander, M., Fürth, D., Karlsson, S., \& Bäckman, L. (2009) Working memory plasticity modulated by dopamine transporter genotype. Neuroscience Letters, 467(2), 117-120. https://doi.org/10.1016/j. neulet.2009.10.018.

Brooks, S. J., Burch, K. H., Maiorana, S. A., Cocolas, E., Schioth, H. B., Nilsson, E. K., Stein, D. J. (2016). Psychological intervention with working memory training increases basal ganglia volume: A VBM study of inpatient treatment for methamphetamine use. NeuroImage: Clinical, 12, 478-491. https://doi.org/10.1016/ j.nicl.2016.08.019.

Buschkuehl, M., Hernandez-Garcia, L., Jaeggi, S. M., Bernard, J. A., \& Jonides, J. (2014) Neural effects of short-term training on working memory. Cognitive, Affective \& Behavioral Neuroscience, 14(1), 147-160. https://doi.org/10.3758/s13415-0130244-9.

Buschkuehl, M., Jaeggi, S. M., \& Jonides, J. (2012). Neuronal effects following working memory training. Developmental Cognitive Neuroscience, 2. https://doi.org/10.1016/j. dcn.2011.10.001.

Chen, G., Cox, R. W., Glen, D. R., Rajendra, J. K., Reynolds, R. C., \& Taylor, P. A. (2019). A tail of two sides: Artificially doubled false positive rates in neuroimaging due to the sidedness choice with t-tests. Human Brain Mapping, 40(3), 1037-1043. https:// doi.org/10.1002/hbm.24399.

Collette, F., Hogge, M., Salmon, E., \& Van der Linden, M. (2006). Exploration of the neural substrates of executive functioning by functional neuroimaging. Neuroscience, 139(1), 209-221. https://doi.org/10.1016/j.neuroscience.2005.05.035.

Colom, R., Martínez, K., Burgaleta, M., Román, F. J., García-García, D., Gunter, J. L., . Thompson, P. M. (2016). Gray matter volumetric changes with a challenging adaptive cognitive training program based on the dual n-back task. Personality and Individual Differences, 98, 127-132. https://doi.org/10.1016/J.PAID.2016.03.087.

Constantinidis, C., \& Klingberg, T. (2016). The neuroscience of working memory capacity and training. Nature Reviews Neuroscience, 17(7), 438-449. https://doi.org/10.1038/ nrn.2016.43.

Dahlin, E., Neely, A. S., Larsson, A., Bäckman, L., \& Nyberg, L. (2008). Transfer of learning after updating training mediated by the striatum. Science (New York, N.Y.), 320(5882), 1510-1512. https://doi.org/10.1126/science.1155466.

Darki, F., \& Klingberg, T. (2015). The Role of Fronto-Parietal and Fronto-Striatal Networks in the Development of Working Memory: A Longitudinal Study. Cerebral Cortex, 25(6), 1587-1595. https://doi.org/10.1093/cercor/bht352.

DeYoung, C. G., Shamosh, N. A., Green, A. E., Braver, T. S., \& Gray, J. R. (2009). Intellect as Distinct From Openness: Differences Revealed by fMRI of Working Memory. Journal of Personality and Social Psychology, 97(5), 883-892. https://doi.org/ 10.1037/a0016615.

Draganski, B., Gaser, C., Busch, V., Schuierer, G., Bogdahn, U., \& May, A. (2004). Changes in grey matter induced by training. Nature, 427,311 . https://doi.org/ 10.1038/427311a.

Draganski, B., Gaser, C., Kempermann, G., Kuhn, H. G., Winkler, J., Büchel, C., \& May, A (2006). Temporal and Spatial Dynamics of Brain Structure Changes during Extensive Learning. The Journal of Neuroscience, 26(23), 6314. http://www.jneurosci.org/cont ent/26/23/6314.abstract.

Durstewitz, D., Seamans, J. K., \& Sejnowski, T. J. (2000). Dopamine-Mediated Stabilization of Delay-Period Activity in a Network Model of Prefrontal Cortex. Journal of Neurophysiology, 83(3), 1733-1750. https://doi.org/10.1152/ jn.2000.83.3.1733.

Edin, F., Klingberg, T., Johansson, P., McNab, F., Tegnér, J., \& Compte, A. (2009). Mechanism for top-down control of working memory capacity. Proceedings of the National Academy of Sciences of the United States of America, 106(16), 6802-6807. https://doi.org/10.1073/pnas.0901894106.

Engvig, A., Fjell, A. M., Westlye, L. T., Moberget, T., Sundseth, Ø., Larsen, V. A., \& Walhovd, K. B. (2010). Effects of memory training on cortical thickness in the elderly. NeuroImage, 52(4), 1667-1676. https://doi.org/10.1016/J. NEUROIMAGE.2010.05.041.

Gaser, C., \& Schlaug, G. (2003a). Brain Structures Differ between Musicians and NonMusicians. The Journal of Neuroscience, 23(27), 9240. http://www.jneurosci.org/ content/23/27/9240.abstract.

Gaser, C., \& Schlaug, G. (2003b). Gray Matter Differences between Musicians and Nonmusicians. Annals of the New York Academy of Sciences, 999(1), 514-517. https:// doi.org/10.1196/annals.1284.062.

Golestani, N., \& Pallier, C. (2006). Anatomical Correlates of Foreign Speech Sound Production. Cerebral Cortex, 17(4), 929-934. https://doi.org/10.1093/cercor/ bhl003.

Golestani, N., Paus, T., \& Zatorre, R. J. (2002). Anatomical Correlates of Learning Novel Speech Sounds. Neuron, 35(5), 997-1010. https://doi.org/10.1016/S0896-6273(02) 00862-0.
Humphreys, G. F., \& Lambon Ralph, M. A. (2015). Fusion and Fission of Cognitive Functions in the Human Parietal Cortex. Cerebral Cortex, 25(10), 3547-3560. https://doi.org/10.1093/cercor/bhu198.

Hyde, K. L., Lerch, J., Norton, A., Forgeard, M., Winner, E., Evans, A. C., \& Schlaug, G. (2009). Musical Training Shapes Structural Brain Development. The Journal of Neuroscience, 29(10), 3019. http://www.jneurosci.org/content/29/10/3019.abstr act.

Ilg, R., Wohlschläger, A. M., Gaser, C., Liebau, Y., Dauner, R., Wöller, A., ... Mühlau, M. (2008). Gray Matter Increase Induced by Practice Correlates with Task-Specific Activation: A Combined Functional and Morphometric Magnetic Resonance Imaging Study. The Journal of Neuroscience, 28(16), 4210. http://www.jneurosci.org/cont ent/28/16/4210.abstract.

Jaeggi, S. M., Buschkuehl, M., Jonides, J., \& Perrig, W. J. (2008). Improving fluid intelligence with training on working memory. Proceedings of the National Academy of Sciences of the United States of America, 105(19), 6829-6833. https://doi.org/ 10.1073/pnas.0801268105.

Jaeggi, S. M., Buschkuehl, M., Perrig, W. J., \& Meier, B. (2010). The concurrent validity of the $N$-back task as a working memory measure. Memory, 18(4), 394-412. https:// doi.org/10.1080/09658211003702171.

Klingberg, T. (2010). Training and plasticity of working memory. Trends in Cognitive Sciences, 14(7), 317-324. https://doi.org/10.1016/j.tics.2010.05.002.

Koenigs, M., Barbey, A. K., Postle, B. R., \& Grafman, J. (2009). Superior parietal cortex is critical for the manipulation of information in working memory. The Journal of Neuroscience : The Official Journal of the Society for Neuroscience, 29(47), 14980-14986. https://doi.org/10.1523/JNEUROSCI.3706-09.2009.

Kühn, S., Schmiedek, F., Noack, H., Wenger, E., Bodammer, N. C., Lindenberger, U., \& Lövden, M. (2013). The dynamics of change in striatal activity following updating training. Human Brain Mapping, 34(7), 1530-1541. https://doi.org/10.1002/ hbm. 22007.

Küper, K., \& Karbach, J. (2016). Increased training complexity reduces the effectiveness of brief working memory training: Evidence from short-term single and dual $\mathrm{n}$-back training interventions. Journal of Cognitive Psychology, 28(2), 199-208. https://doi. org/10.1080/20445911.2015.1118106.

Lawlor-Savage, L., Clark, C. M., \& Goghari, V. M. (2019). No evidence that working memory training alters gray matter structure: A MRI surface -based analysis. Behavioural Brain Research, 360, 323-340. https://doi.org/10.1016/j. bbr.2018.12.008.

Mårtensson, J., Eriksson, J., Bodammer, N. C., Lindgren, M., Johansson, M., Nyberg, L., \& Lövdén, M. (2012). Growth of language-related brain areas after foreign language learning. NeuroImage, 63(1), 240-244. https://doi.org/10.1016/J. NEUROIMAGE.2012.06.043.

McNab, F., Varrone, A., Farde, L., Jucaite, A., Bystritsky, P., Forssberg, H., \& Klingberg, T. (2009). Changes in Cortical Dopamine D1 Receptor Binding Associated with Cognitive Training. Science, 323(5915), 800-802. https://doi.org/10.1126/ science.1166102.

McNab, Fiona, \& Klingberg, T. (2008). Prefrontal cortex and basal ganglia control access to working memory. Nature Neuroscience, 11(1), 103-107. https://doi.org/10.1038/ nn2024.

Metzler-Baddeley, C., Caeyenberghs, K., Foley, S., \& Jones, D. K. (2016). Task complexity and location specific changes of cortical thickness in executive and salience networks after working memory training. NeuroImage, 130, 48-62. https://doi.org/10.1016/j. neuroimage.2016.01.007.

Miró-Padilla, A., Bueichekú, E., \& Ávila, C. (2020). Locating neural transfer effects of nback training on the central executive: A longitudinal fMRI study. Scientific Reports, 10(1), 5226. https://doi.org/10.1038/s41598-020-62067-y.

Miró-Padilla, A., Bueichekú, E., Ventura-Campos, N., Flores-Compañ, M.-J., Parcet, M. A., \& Ávila, C. (2019). Long-term brain effects of N-back training: An fMRI study. Brain Imaging and Behavior, 13(4), 1115-1127. https://doi.org/10.1007/ s11682-018-9925-x.

Nee, D. E., Brown, J. W., Askren, M. K., Berman, M. G., Demiralp, E., Krawitz, A., \& Jonides, J. (2013). A Meta-analysis of Executive Components of Working Memory. Cerebral Cortex, 23(2), 264-282. https://doi.org/10.1093/cercor/bhs007.

Owen, A. M., McMillan, K. M., Laird, A. R., \& Bullmore, E. (2005). N-back working memory paradigm: A meta-analysis of normative functional neuroimaging studies Human Brain Mapping, 25(1), 46-59. https://doi.org/10.1002/hbm.20131.

Owens, M. M., Duda, B., Sweet, L. H., \& MacKillop, J. (2018). Distinct functional and structural neural underpinnings of working memory. NeuroImage, 174(December 2017), 463-471. https://doi.org/10.1016/j.neuroimage.2018.03.022.

Palomar-García, M.Á., Zatorre, R. J., Ventura-Campos, N., Bueichekú, E., \& Ávila, C. (2017). Modulation of Functional Connectivity in Auditory-Motor Networks in Musicians Compared with Nonmusicians. Cerebral Cortex, 27(5), 2768-2778. https://doi.org/10.1093/cercor/bhw120.

Salminen, T., Kühn, S., Frensch, P. A., \& Schubert, T. (2016). Transfer after dual n-back training depends on striatal activation change. Journal of Neuroscience, 36(39), 10198-10213. https://doi.org/10.1523/JNEUROSCI.2305-15.2016.

Schneiders, J. a, Opitz, B., Krick, C. M., \& Mecklinger, A. (2011). Separating intra-modal and across-modal training effects in visual working memory: an fMRI investigation. Cerebral Cortex (New York, N.Y. : 1991), 21(11), 2555-2564. https://doi.org/ 10.1093/cercor/bhr037.

Sluming, V., Barrick, T., Howard, M., Cezayirli, E., Mayes, A., \& Roberts, N. (2002). Voxel-Based Morphometry Reveals Increased Gray Matter Density in Broca's Area in Male Symphony Orchestra Musicians. NeuroImage, 17(3), 1613-1622. https://doi. org/10.1006/NIMG.2002.1288.

Söderqvist, S., Matsson, H., Peyrard-Janvid, M., Kere, J., \& Klingberg, T. (2014). Polymorphisms in the Dopamine Receptor 2 Gene Region Influence Improvements 
during Working Memory Training in Children and Adolescents. Journal of Cognitive Neuroscience, 26(1), 54-62. https://doi.org/10.1162/jocn_a_00478.

Söderqvist, S., Nutley, S. B., Peyrard-Janvid, M., Matsson, H., Humphreys, K., Kere, J., \& Klingberg, T. (2012). Dopamine, working memory, and training induced plasticity: Implications for developmental research. Developmental Psychology, 48(3), 836-843. https://doi.org/10.1037/a0026179.

Soveri, A., Antfolk, J., Karlsson, L., Salo, B., \& Laine, M. (2017). Working memory training revisited: A multi-level meta-analysis of n-back training studies. Psychonomic Bulletin \& Review. https://doi.org/10.3758/s13423-016-1217-0.

Stein, M., Federspiel, A., Koenig, T., Wirth, M., Strik, W., Wiest, R., ... Dierks, T. (2012). Structural plasticity in the language system related to increased second language proficiency. Cortex, 48(4), 458-465. https://doi.org/10.1016/J.

CORTEX.2010.10.007.
Takeuchi, H., Taki, Y., \& Kawashima, R. (2010). Effects of working memory training on cognitive functions and neural systems. Reviews in the Neurosciences, 21(6), 427-449. http://europepmc.org/abstract/MED/21438192.

Takeuchi, H., Taki, Y., Sassa, Y., Hashizume, H., Sekiguchi, A., Fukushima, A., \& Kawashima, R. (2011). Working memory training using mental calculation impacts regional gray matter of the frontal and parietal regions. PLOS ONE, 6(8). https://doi. org/10.1371/journal.pone.0023175.

Ullman, H., Almeida, R., \& Klingberg, T. (2014). Structural Maturation and Brain Activity Predict Future Working Memory Capacity during Childhood Development. Journal of Neuroscience, 34(5), 1592-1598. https://doi.org/10.1523/JNEUROSCI.084213.2014.

Zou, Q., Ross, T. J., Gu, H., Geng, X., Zuo, X.-N., Hong, L. E.a., Zang, Y.-F., \& Yang, Y (2013). Intrinsic resting-state activity predicts working memory brain activation and behavioral performance. Human Brain Mapping, 34(12), 3204-3215. https://doi.org/ $10.1002 / \mathrm{hbm} .22136$ 